\title{
An Innovative Model for the Industrial Chain of the Internet and Traditional Culture
}

\author{
Wen Ying ${ }^{1}$ and $\mathrm{Li} \mathrm{Hao}{ }^{2}$ \\ 1. Changsha University of Science \& Technology. \\ 2. Hunan zhongyi communication technology Engineering Co., Ltd. \\ E-mail: 395208367@qq.com
}

\begin{abstract}
In allusion to the insufficient industrialization incentive of the existing policy for NEV (New-Energy-Vehicle), an incentive mechanism based on preferential tax rate is proposed in this paper to establish the corresponding NEV industrialization system dynamics model. Meanwhile, the model is simulated and analyzed on the basis of such state indexes as market expansion degree to give the reference values of the two incentive tools in different periods, namely: the preferential tax rate for NEW manufacturing industry and the income tax rate additionally levied on the traditional vehicle manufacturing industry. The simulation result shows that the incentive mechanism can expand NEV consumer market but will not reduce the corporate income tax for the national finance. Compared with the indexes under single policy, various state indexes are improved to present obvious incentive effect. Therefore, such research can provide new thought and method for incentive mechanism design.
\end{abstract}

Keywords: Preferential tax rate; System dynamics; Incentive mechanism; NEV; Industrialization

\section{Introduction}

As an important part of the strategic emerging industry, NEV industry in China is still in the initial industrialization stage. Powerful and effective national policies are essential to the large-scale production [1]. At present, NEV has such problems as high cost, high price and low economic benefit, but NEV enterprises still pursue for profit maximization. Under such situation, it is necessary to give play to the leading effect of the government behaviors so as to motivate the production enthusiasm of NEV enterprises. During NEV industrialization process, the capital source for policy implementation should not be ignored. According to the Vehicle Industry Adjustment and Promotion Planning issued in March 2009, NEV sales volume will occupy about $5 \%$ of the passenger vehicle sales volume in 2015 . It is predicted that the passenger vehicle sales volume in 2015 in China would be 13,500,000 sets and NEV sales volume would exceed 600,000 sets. If each NEV is subsidized with RMB 30,000 40,000 Yuan, then the total subsidy will reach up to RMB $18,000,000,000 \sim 24,000,000,000$ Yuan. Obviously, it is very difficult for the national finance department to raise such subsidy capitals.

In conclusion, it is necessary to design NEV industrialization incentive mechanism based on preferential tax rate so as to give play to the advantages of the preferential tax rate and meanwhile conquer the disadvantages during the implementation process, thus to maximally solve the capital source problem. From a systematical angle, an incentive mechanism based on preferential tax rate is proposed in this paper on the principle of incentive and restriction uniformity, and the capital source problem of the incentive mechanism implementation is also considered. According to the simulation result of NEV industrialization system dynamics model, the incentive effect of the incentive mechanism is obviously superior to the single policy. 


\section{Incentive Mechanism based on Preferential Tax Rate}

Incentive purpose is an essential part of the incentive mechanism design, and the incentive purpose of the incentive mechanism designed in this paper includes the following two aspects:

1) Rapidly promote NEV industrialization: through the incentive mechanism implementation, various state indexes of NEV industrialization can be significantly improved;

2) Solve the capital source problem for the government during the incentive mechanism implementation process, without reducing relevant national tax revenues: the incentive mechanism based on preferential tax rate can balance the preferential tax revenues for NEV manufacturers and the tax revenues additionally levied on traditional vehicle manufacturers in order to promote NEV industrialization development while not reducing relevant national tax revenues.

For above incentive purpose and principle, NEV industrialization incentive mechanism based on preferential tax rate is designed in this paper:

1) The incentive subject is the government, and the incentive object is NEV manufacturers and traditional vehicle manufacturers.

2) Incentive function: preferential corporate income tax rate $X \%$ is given to NEV manufacturers. The total preferential income taxes for each year $M(100,000,000$ Yuan/year) $=X \% \times \mathrm{NEV}$ sales volume $(100,000,000$ Yuan/year $)$

3) Restraint function: levied corporate income tax rate $Y \%$ is given to traditional vehicle manufacturers. The total corporate income taxes additionally levied $N(100,000,000$ Yuan/year $)=Y \% \times$ traditional vehicle sales volume $(100,000,000$ Yuan/year $)$

\section{System Dynamics Model}

\subsection{Establishment of NEV Industrialization SD Model}

SD (System Dynamics) is a system approach combining qualitative and quantitative researches for handling complex problems. Specifically, feedback control theory, information theory, system theory, decision theory, computer simulation and system analysis method are integrated in SD in order to systematically analyze and solve problems, describe the dynamical complexity of the system and assist people to understand the structural causes of dynamical behaviors of the system through cause \& effect feedback relationship. Therefore, this method is especially applicable to such nonlinear complex large-scale system problems as social problem, economic problem and ecological problem.

Vensim PLE 5.6a is adopted in this paper to establish NEV industrialization SD model. Viewed from the aspects of system operation and upstream \& downstream, NEV industrialization includes three sub-systems, namely industrialization investment sub-system, industrialization process sub-system and industrialization benefit sub-system, wherein NEV industrialization investment is selected from the industrialization investment sub-system; NEV industrial accumulative output, NEV accumulative sales volume and NEV new markets are selected from the industrialization process sub-system; NEV industrialization benefit, environmental benefit, energy benefit and other key variables are selected from the industrialization benefit sub-system. Intuitively, the causal graph formed by these variables is as shown in Figure 1. 


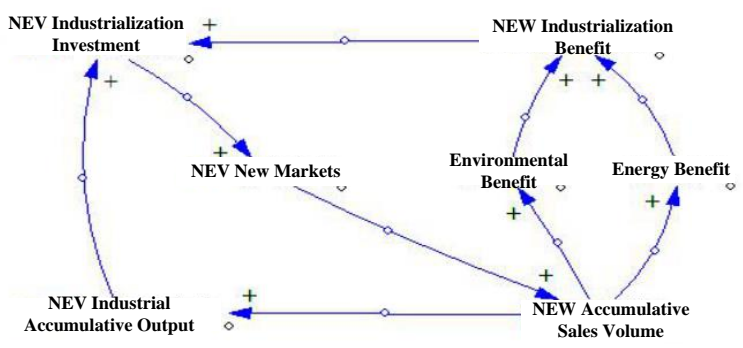

Figure 1. Key Variable Causal Graph

According to Figure 1, NEV industrialization investment increase and the incentive mechanism implementation can expand NEV new markets and increase NEV accumulative sales volume. Meanwhile, NEV industrial accumulative output and NEV industrialization benefit brought thereby are correspondingly continuously increased, and they also have positive feedback effect on NEV industrialization investment to promote the further increase thereof.

\subsection{Model Rationality Verification}

\subsubsection{Structural Rationality Verification}

A lot of relevant literatures, data issued by relevant enterprises, statistical yearbooks for vehicle industry, relevant research reports and other statistical information \& network resources are adopted in this paper for the system analysis and the model establishment of NEV industrialization. Through in-depth research and consultation to relevant professional personnel, the system dynamics model structure is basically ensured to be consistent with NEV industrialization practice.

\subsubsection{Model Output Data Verification}

Through comparing the basic output data of the model with the data issued by relevant national departments and associations, we believe that the output result of the proposed model is basically consistent with the practical situation of NEV industrial development in China, and the historical data collection can meet the basic requirements for model rationality.

\section{Simulation Experiment}

\subsection{State Index}

In this paper, the state indexes of NEV industrialization are established according to the following five aspects:

1) Market expansion degree: the market expansion degree is mainly presented in market shares and customer liquidity. In this paper, the proportion of annual NEV sales volume in total annual vehicle sales volume $N X L B$ is regarded as the measurement index of the market expansion degree, and the target value is $M N X L B$.

2) Investment domination degree: when NEV industry gradually becomes dominant, the investments from the whole society will be gradually increased. In this paper, the proportion of NEV industrialization investment in the total investment for the vehicle industry $K B$ is regarded as the measurement index for the industry domination degree, and the target value is $M K B$.

3) Industrial economic benefit: enterprises are the market subject for seeking profits, and profits are the economic source power of the enterprise development. In this paper, NEV 
industrial profit rate $C Y L R L$ is regarded as the measurement index for the industrial economic benefit, and the target value is MCYLRL.

4) Energy benefit: energy conservation is an important strategic target for a country to develop NEV industry. Along with the development of NEV industrialization, the consumption of such resources as fuel oil and natural gas will be reduced, and the economic benefit brought thereby is namely the energy benefit. In this paper, $N Y X Y$ represents the energy benefit, and the target value is $M N Y X Y$.

5) Environmental benefit: the environmental benefit refers to the benefit brought by NEV industrialization development for reducing such harmful gases as NOx and carbon monoxide.

The specific values of various targets are determined according to the Energy Conservation and NEV Industry Planning (2011-2020) and relevant literatures. Please refer to Table 1. Data source [18]

Table 1. State Index and Target Value

\begin{tabular}{cccc}
\hline Target & State Index & Unit & Target Value for Year 2020 \\
\hline MNYXY & Energy benefit & $100,000,000$ Yuan & 1000 \\
MHJXY & Environmental benefit & $00,000,000$ Yuan & 10 \\
MNXLB & Market expansion degree & Percentage & $15 \%$ \\
MKB & Industry domination degree & Percentage & $15 \%$ \\
MCYLRL & Profit rate of NEV industry & Percentage & $20 \%$ \\
\hline
\end{tabular}

\subsection{Simulation and Effect Evaluation}

In order to analyze and compare the effects of the incentive mechanism based on preferential tax rate and the single policy on NEV industry development, the following three simulation schemes are designed in this paper.

Simulation scheme 1: NEV incentive mechanism based on preferential tax rate is introduced, and NEV preferential tax rate coefficient is set as 1, and BaseRun is set as the simulation database.

Simulation scheme 2: under the condition that the income tax rate levied on the traditional vehicle manufacturing industry is not introduced therein as the restraint, namely only under the effect of the single policy, NEV preferential tax rate coefficient is set as 1.5, indicating that the government yearly increases the preferential tax rate for NEV manufacturing industry by $50 \%$, and other parameters are not changed, and Test 1 is set as the simulation database.

Simulation scheme: under the condition that the income tax rate levied on the traditional vehicle manufacturing industry is not introduced therein as the restraint, namely only under the effect of the single policy, NEV preferential tax rate coefficient is set as 0.5 , indicating that the government yearly reduces the preferential tax rate for NEV manufacturing industry by $50 \%$, and other parameters are not changed, and Test 2 is set as the simulation database.

In the following simulation and result analysis, we will simulate and analyze the variation of the preferential tax rate for NEV manufacturing industry and the income tax rate levied on traditional vehicle manufacturing industry after the incentive mechanism is introduced, and mainly focus on the influence of each state index (market expansion index, industry domination index, industrial economic benefit, energy benefit and environment benefit) on NEV industrialization. 


\subsubsection{Variation of Preferential Tax Rate for NEV Manufacturing Industry}

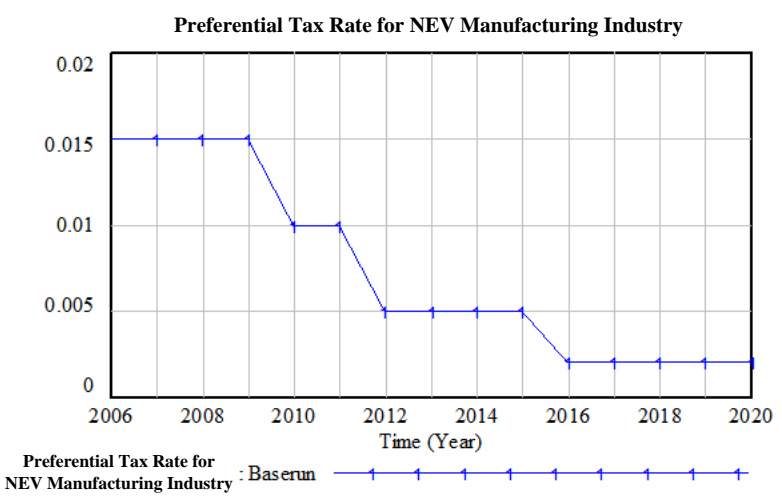

(a)

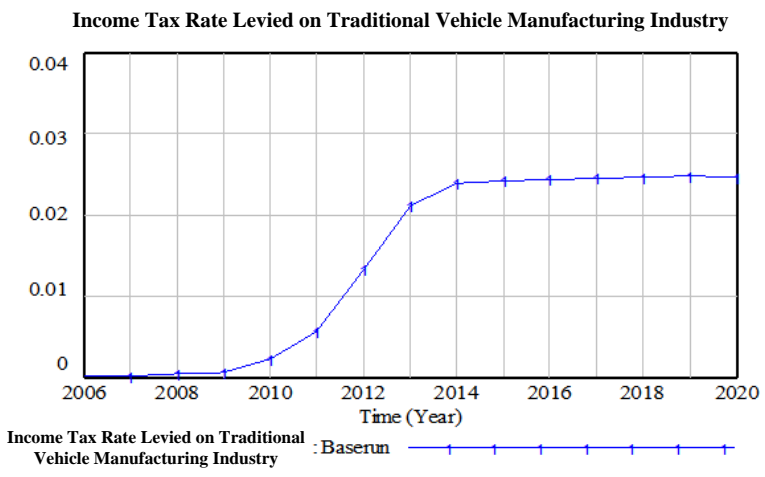

(b)

Figure 2. Variation of Preferential Tax Rate for Nev Manufacturing Industry and Income Tax Rate Levied on Traditional Vehicle Industry

As shown in Figure 2(a), the preferential tax rate for NEV manufacturing industry is reduced year by year, and this is consistent with the practical situation, because the profits of NEV manufacturers are increased along with NEV cost reduction and the preferential tax rate needed thereby correspondingly tends to be reduced.

\subsubsection{Variation of Income Tax Rate Levied on Traditional Vehicle Manufacturing Industry}

As shown in Figure 2(b), the maximum income tax rate levied on traditional vehicle manufacturing industry during 2006 2020 is $2.4 \%$, and compared with the present income tax rate levied on traditional vehicle manufacturers ---- $2.5 \%$, such income tax rate is not significantly changed and can be accepted by enterprises. 
4.2.3. Comparison of Proportions (market expansion index) of NEV Sales Volume in Total Vehicle Sales Volume in the Three Schemes

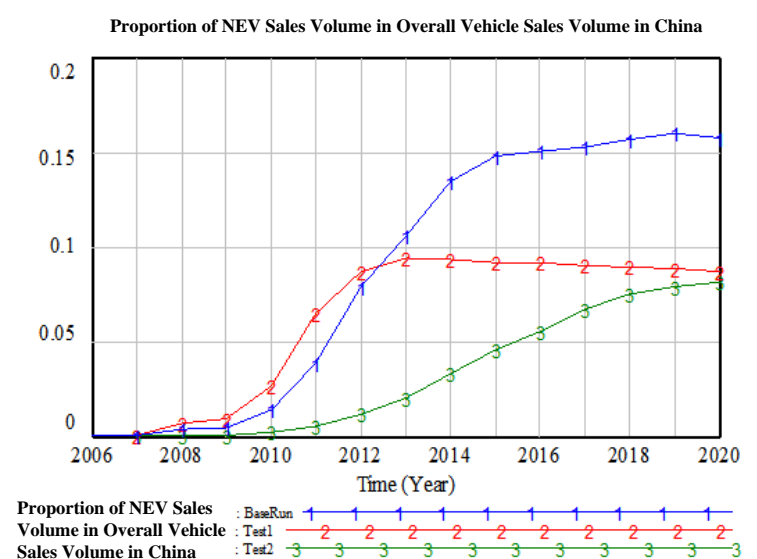

(a)

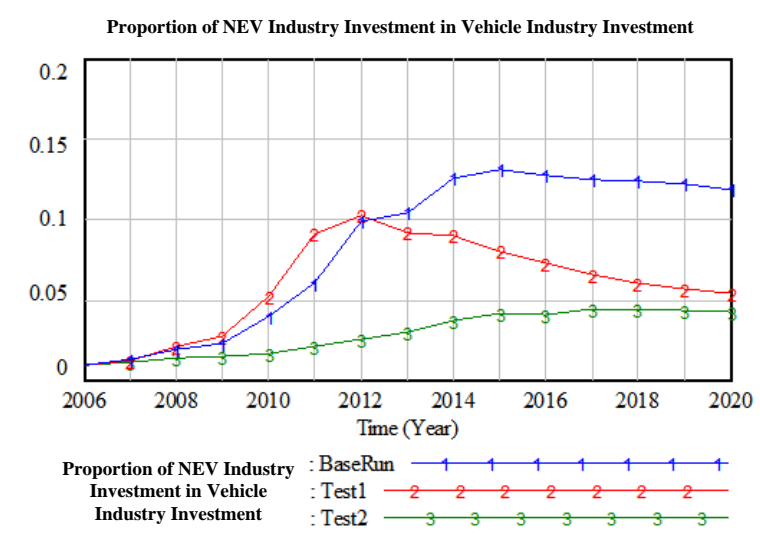

(b)

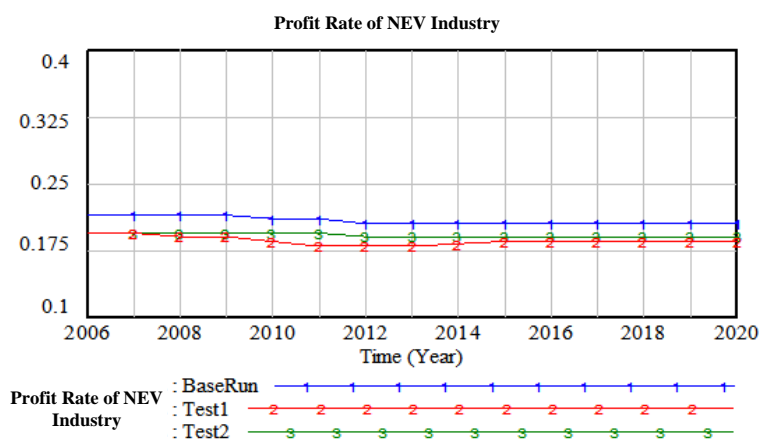

(c)

\section{Figure 3. Comparison of Market Expansion Index, Industry Domination Index and Industrial Economic Benefit in Three Schemes}

According to Figure 3(a), due to the incentive mechanism implementation, the proportion of NEV annual sales volume in the overall annual vehicle sales volume can reach up to $15 \%$ of the target value of the market expansion degree index in 2016, and this proportion will be fluctuated in a small range. This indicates that the incentive mechanism has dual attributes -- incentive and restraint, and compared with the single policy, this incentive mechanism has 
better incentive effect for the incentive object, stronger market expansion ability, and stronger promotion for NEV industrialization.

Viewed from the effect time of the incentive mechanism, the incentive mechanism is initially implemented during 2006 2010, NEV market is slowly expanded; along with the gradually strengthened restraint of the incentive mechanism on traditional vehicle manufacturers, the proportion of NEV annual sales volume in the overall vehicle sales volume in China has the rapidest increase during 2010 2014, thus indicating that NEV market expansion ability of this incentive mechanism becomes highlighted and is being gradually strengthened.

\subsubsection{Comparison of Proportions of NEV Industry Investment in Vehicle Industry Investment (industry domination index) in Three Schemes}

As shown in Figure 3(b), the government investment is the main investment in the initial industrialization period, so line 2 is above line 1, but along with the gradual appearance of the incentive mechanism implementation effect, NEV sales volumes is rapidly increased, and the enterprise investment gradually replaces the government investment and becomes the investment subject. Line 1 is above line 2 in 2012 for the first time, and then gradually rises up to the peak $12.2 \%$ around 2015 , and finally becomes stable, thus indicating that the investment enthusiasm and intensity of the enterprises are changed along with the market enthusiasm.

\subsubsection{Comparison of NEV Industrial Profit Rates (industrial economic benefit index) in Three Schemes}

According to Figure 3(c), compared with other two schemes, the incentive mechanism can more or less improve NEV industrial profit rate after implementation. Specifically, the minimum value is $20.5 \%$ and namely reaches $20 \%$ of the target value of the industrial economic benefit, thus indicating that the incentive mechanism based on preferential tax rate directly acts on NEV manufacturing industry and presents obvious incentive effect.

\subsubsection{Comparison of Energy Benefit and Environmental Benefit in Three Schemes}

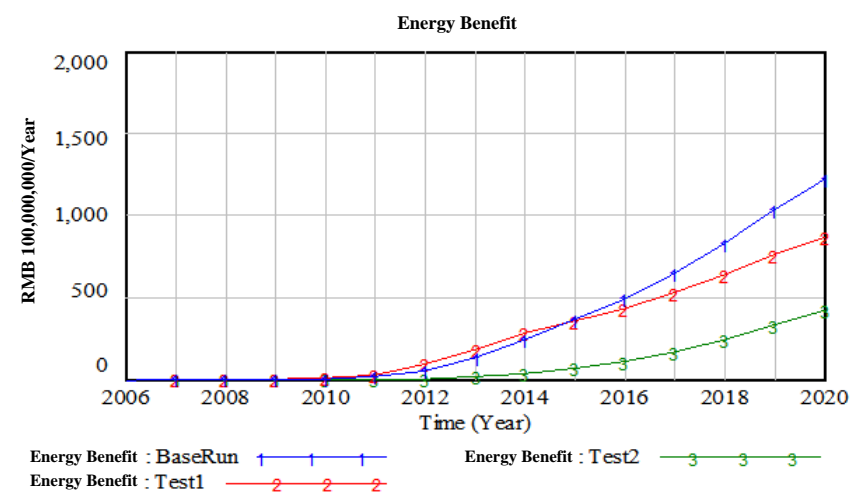

(a) 


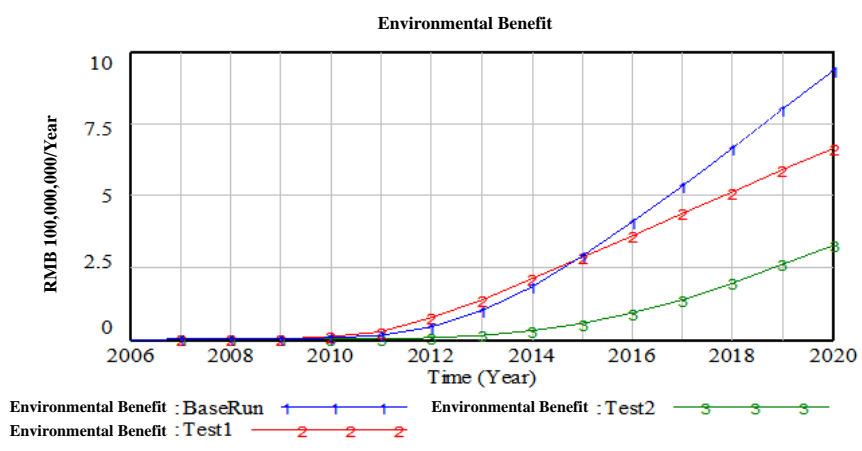

(b)

\section{Figure 4. Comparison of Energy Benefits and Environmental Benefits in Three Schemes}

According to Figures 4(a) and 4(b), along with the incentive mechanism implementation, NEV accumulative sales volume is increased year by year, the energy benefit and the environmental benefit brought thereby are rapidly increased. Line 1 is above line 2 around 2015 when NEV sales volume is rapidly increased. Finally in 2020, the energy benefit will be RMB 121,800,000,000 Yuan, exceeding the target value (RMB 100,000,000,000 Yuan), and the environmental benefit will be realized as RMB 937,000,000 Yuan, slightly below the target value (RMB 1,000,000,000 Yuan)

\section{Conclusion}

The NEW industrialization incentive mechanism based on preferential tax rate is designed in this paper, and the corresponding system dynamics model is established for the incentive mechanism simulation in order to give the reference values of the two incentive tools, namely the preferential tax rate for NEV manufacturing industry and the income tax rate levied on the traditional vehicle manufacturing industry. The simulation result shows: due to NEV price advantage relatively to the traditional vehicles after the incentive mechanism implementation, the new markets influenced by price factors can be significantly expanded, thus to obviously improve the state indexes of NEV industrialization and present the obvious incentive effect of the incentive mechanism. Additionally, the incentive mechanism based on preferential tax rate can really act on NEV new markets only after long-term chain and system feedbacks, so the incentive mechanism needs to take long time to give play to its functions.

\section{Acknowledgements}

Fund project of Hunan Province philosophy and social sciences research and heritage digital protection of Hunan folk paper-cut art from the perspective of the intangible cultural heritage(13JL02) and Hunan Provincial Department of Education Fund Project: Hunan folk paper-cut art digital protection technology research(15C0067).

\section{References}

[1] Xiaolei Zhang, Yong Han, Dongsheng Hao and Zhihan Lv. ARPPS: Augmented Reality Pipeline Prospect System. 22th International Conference on Neural Information Processing (ICONIP 2015), Istanbul, Turkey. In press.

[2] Wei Gu, Zhihan Lv, Ming Hao. Change detection method for remote sensing images based on an improved Markov random field. Multimedia Tools and Applications. 2016.

[3] Zhihan Lu, Chantal Esteve, Javier Chirivella and Pablo Gagliardo. A Game Based Assistive Tool for Rehabilitation of Dysphonic Patients. 3rd International Workshop on Virtual and Augmented Assistive Technology (VAAT) at IEEE Virtual Reality 2015 (VR2015), Arles, France, IEEE, 2015. 
[4] Zhihan Lv, Alaa Halawani, Shengzhong Feng, Haibo Li, and Shafiq Ur Rehman. Multimodal Hand and Foot Gesture Interaction for Handheld Devices. ACM Transactions on Multimedia Computing, Communications, and Applications (TOMM). 11, 1s, Article 10 (October 2014), 19 pages.

[5] Yancong Lin, Jiachen Yang, Zhihan Lv, Wei Wei, Houbing Song. A Self-Assessment Stereo Capture Model Applicable to the Internet of Things. Sensors. 2015.

[6] Wei Ou, Zhihan Lv, Zanfu Xie. Spatially Regularized Latent topic Model for Simultaneous object discovery and segmentation. The 2015 IEEE International Conference on Systems, Man, and Cybernetics (SMC2015).

[7] Yi Wang, Yu Su, Gagan Agrawal. A Novel Approach for Approximate Aggregations Over Arrays. In Proceedings of the 27th international conference on scientific and statistical database management, ACM, 2015.

[8] Zhihan Lv, Alaa Halawani, Shengzhong Feng, Shafiq ur Rehman, Haibo Li. Touch-less Interactive Augmented Reality Game on Vision Based Wearable Device. Personal and Ubiquitous Computing. 2015.

[9] Jiachen Yang, Shudong He, Yancong Lin, Zhihan Lv. Multimedia cloud transmission and storage system based on internet of things. Multimedia Tools and Applications. 2016.

[10] Dingde Jiang, Xu Ying, Yang Han, Zhihan Lv. Collaborative Multi-hop Routing in Cognitive Wireless Networks. Wireless Personal Communications. 2015.

[11] Weixi Wang, Zhihan Lu, Xiaoming Li, Weiping Xu, Baoyun Zhang, Xiaolei Zhang. Virtual Reality Based GIS Analysis Platform. 22th International Conference on Neural Information Processing (ICONIP 2015), Istanbul, Turkey.

[12] Zhihan Lv, Chantal Esteve, Javier Chirivella and Pablo Gagliardo. Clinical Feedback and Technology Selection of Game Based Dysphonic Rehabilitation Tool. 2015 9th International Conference on Pervasive Computing Technologies for Healthcare (PervasiveHealth2015), IEEE, 2015.

[13] Jiachen Yang et al. A Low-Power and Portable Biomedical Device for Respiratory Monitoring with a Stable Power Source. Sensors 15.8 (2015): 19618-19632.

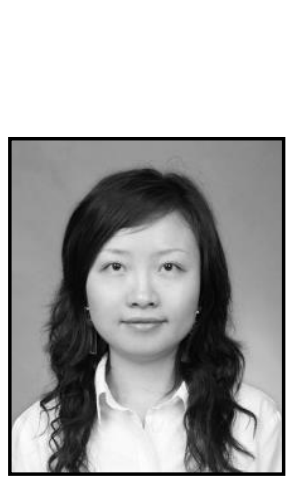

\section{Authors}

Wen Ying, She received her M.S. degree in agricultural extension from Central South University of Forestry and Technology in Changsha, China. She is currently a lecturer in the College of Art \& Design at Changsha university of Science \&Technology. Her research interest is mainly in the area of cultural industry chain upgrading, Interaction design. She has published several research papers in scholarly journals in the above research areas and has participated in several books. 
International Journal of $\mathrm{u}-$ and $\mathrm{e}-\mathrm{Service}$, Science and Technology Vol. 10, No. 2 (2017) 